\title{
Optical Studies of the Correlation Between Interface Disorder and the Photoluminescence Line Shape in GaAs/InGaP Quantum Wells
}

\author{
E. Laureto, E. A. Meneses, \\ Instituto de Física "Gleb Wataghin", Universidade Estadual de Campinas, 13083-970 Campinas - SP, Brazil \\ W. Carvalho Jr., A. A. Bernussi, E. Ribeiro, \\ Laboratório Nacional de Luz Síncrotron, 13088-902 Campinas - SP, Brazil \\ E. C. F. da Silva, \\ Instituto de Física da Universidade de São Paulo, 05508-900, São Paulo - SP, Brazil \\ and J. B. B. de Oliveira \\ Departamento de Física, Universidade Estadual Paulista, \\ Av. Eng. Luis Edmundo C. Coube, s/n, 17033 - 360, Bauru - SP, Brazil
}

Received on 23 April, 2001

\begin{abstract}
Photoluminescence (PL) and excitation PL measurements have been performed at different temperatures in a number of lattice-matched $\mathrm{GaAs} / \mathrm{In}_{0.49} \mathrm{Ga}_{0.51} \mathrm{P}$ quantum wells, where the fluctuations of the potential energy are comparable with the thermal energy of the photocreated carriers. Two samples with different well widths allow to observe a series of anomalous effects in their optical response. The observed effects are related to the disorder in the interface, characterizing fluctuations in the confinement potential energy. It is proposed that the carrier relaxation processes occur either at the local minima or at the absolute minimum of the confinement potential, depending on the ratio of the thermal energy and the magnitude of the potential fluctuations.
\end{abstract}

\section{Introduction}

Semiconductor heterostructures like quantum wells (QW), quantum wires and quantum dots, have been extensively investigated in the last decade, since they are extremely important for the high performance of the electronic and optoelectronic devices [1]. In these systems the excitonic properties are strengthened due to the carriers confinement potential $[2,3]$. In the QW case, variations in the quantum well width and alloy chemical compositions cause localized fluctuations in this potential. Such fluctuations modulate the local profile of the valence and conduction bands, then affecting the optical properties of semiconductor heterostructures. Optical spectroscopy such as photoluminescence (PL) and excitation PL (PLE) are powerful tools for investigating these properties. The excitons created by optical absorption relax to lower energy states before radiative recombination. Therefore $\mathrm{PL}$ and PLE together can give information about the confinement potential fluctuations through the peak position and the line shape of excitonic spectra.

In this work we study the influence of the fluctuations of the confinement potential on the optical prop- erties of the excitons in GaInP/GaAs quantum wells. Similar results were observed by Grassi Alessi et al. [3]for InGaAs/GaAs quantum wells. We have used two single quantum well samples with the same growing conditions, but different nominal QW widths. PL and PLE spectra were taken at $2 \mathrm{~K}$ and as a function of temperature up to $100 \mathrm{~K}$. We show that the shape and the temperature dependence of the PL lines are governed by the exciton energy relaxation. A qualitative fluctuation potential model is proposed where the carrier relaxation processes occur either at the local minima or at the absolute minimum of the confinement potential, depending on the ratio of the thermal energy and the magnitude of the potential fluctuations.

\section{Experimental Results}

The samples used in this study were grown on GaAs (100)-oriented substrates by metal-organic chemical vapor deposition (MOCVD) at $580{ }^{\circ} \mathrm{C}$. The samples consist of a GaAs buffer, a $500 \AA$ GaInP barrier, a GaAs layer of width $\mathrm{L}_{Q W}$ and another $500 \AA$ GaInP layer. In order to avoid chemical reactions, in the first 
GaAs/GaInP interface a GaP layer was grown during 1 sec. The samples are nominally undoped and the measured lattice mismatch is less than $10^{-3}$. The optical measurements were done in a He immersion cryostat using a Ti-sapphire laser as excitation source (with energy fixed in $1.71 \mathrm{eV}$ for PL measurements) and excitation intensity of $\sim 0.5 \mathrm{~W} / \mathrm{cm}^{2}$ for all spectra. The luminescence was detected and analyzed by a standard lock-in technique. The PLE spectra were detected in the low energy side of PL curve in order to optimize the e1-hh1 transition line shape. In Table I we list relevant information about the two samples, from now on referred to as sample A and sample B.

\begin{tabular}{ccccc}
\hline \hline Sample & $\mathrm{L}_{Q W}(\AA)$ & FWHM $_{P L E}$ & FWHM $_{P L}$ & SS \\
\hline $\mathrm{A}$ & 80 & 3.8 & 3.6 & 7.4 \\
$\mathrm{~B}$ & 30 & 11.5 & 8.4 & 19.4 \\
\hline \hline
\end{tabular}

TABLE I: QW width $\left(\mathrm{L}_{Q W}\right)$, FWHM for PLE and PL, and the measured Stokes Shift (SS) for samples A and B. Experimental values are given in meV (uncertainties of about $5 \%$ ) and were taken at $2 \mathrm{~K}$.

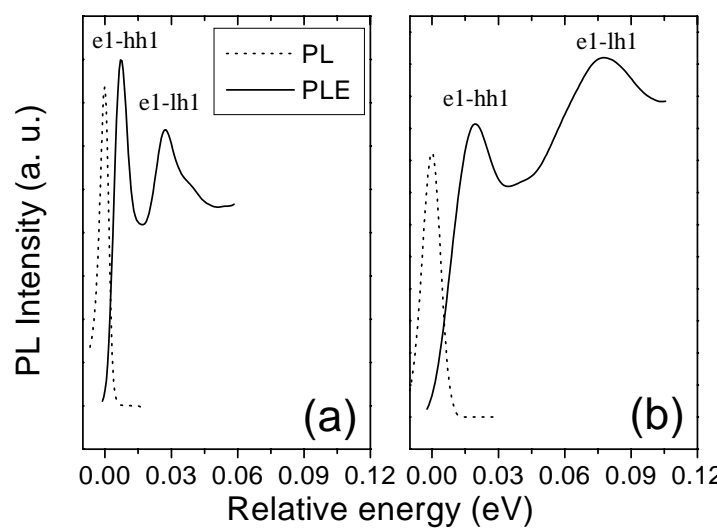

Figure 1. PL (dotted line) and PLE (continuous line) spectra for samples (a) A and (b) B. Measurements were done at $2 \mathrm{~K}$. The energy scale is redefined so that the zero corresponds to the PL peak energy for each sample.

In Fig. 1 we show the PL (dotted line) and PLE (continuous line) spectra at $2 \mathrm{~K}$ for samples A [Fig. 1(a)] and B [Fig. 1(b)]. In this figure the energy scale was adjusted so that the zero energy corresponds to the position of the intensity maximum of the PL line. The objective of this choice is to emphasize the difference between the positions of the energy maximum of PL and PLE spectra (Stokes shift, SS) and the full width at half maximum of PL and PLE spectra (FWHM). The PLE spectra show the absorption resonances of the heavy hole (e1-hh1) and light hole (e1-lh1) excitons. We can see in Fig. 1 that the distance $\Delta \mathrm{E}$ between these two peaks is larger in sample $\mathrm{B}$ than in sample $\mathrm{A}$, in qualitative agreement with the energy levels as a function of $\mathrm{L}_{Q W}$, calculated by an effective-mass model $(\Delta \mathrm{E} \simeq$ $1 / \mathrm{L}_{Q W}$ ) [4]. We can also observe that the ratio between the e1-lh1 and e1-hh1 peak intensities in PLE spectra is inverted in Fig. 1(a) and 1(b). Similar effect was observed by Jahn et al [5]. They associated the reduction of PLE resonance intensity relative to the heavy hole (e1-hh1) to strong localization of recombining excitons in the potential fluctuations. The $\mathrm{FWHM}_{P L E}$, FWHM $_{P L}$ and the SS values obtained from Fig.1 are presented in Table I. We can see in this Table that sample B presents $\mathrm{FWHM}_{P L E}, \mathrm{FWHM}_{P L}$ and SS values greater than the corresponding ones for sample A.

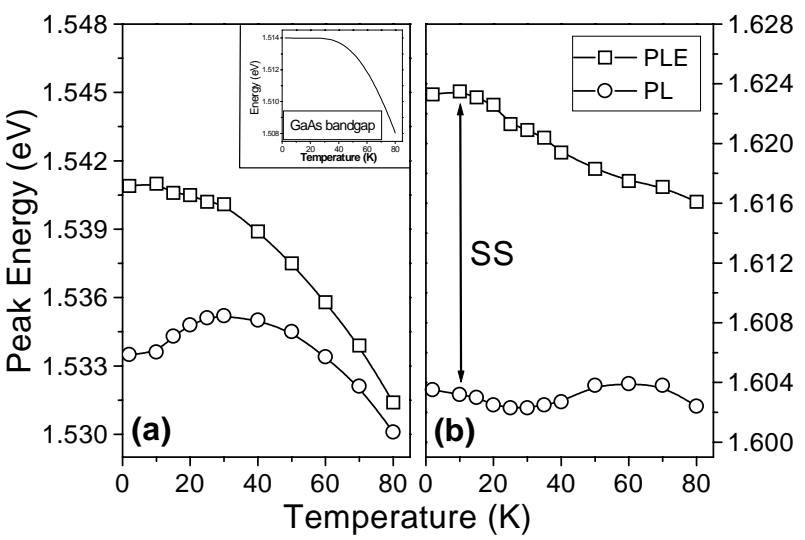

Figure 2. PLE (squares) and PL (circles) peak energy as a function of the temperature for samples (a) A and (b) B. Continuous lines are only a guide to the eyes. Inset presents the GaAs band gap dependence with the temperature.

In Fig. 2 we show the variation with the temperature of the energy peak position for PL (circles) and PLE (squares) spectra for sample A [Fig. 2(a)] and sample B [Fig. 2(b)]. This provides the temperature dependence of the Stokes shift (SS) as indicated at $10 \mathrm{~K}$ by the double-headed arrow in Fig. 2(b). The PLE peak energy decreases with increasing temperature roughly following the GaAs energy band gap [inset in Fig. 2(a)]. On the contrary, the dependence on $\mathrm{T}$ of $\mathrm{PL}$ peak energy exhibits an anomalous behaviour, more pronounced in sample $B$ where the behaviour is sigmoid, nonmonotonic.

In Fig. 3 we plot the dependence of $\mathrm{FWHM}_{P L}$ on temperature for samples $\mathrm{A}$ and $\mathrm{B}$. We observe that for sample A the $\mathrm{FWHM}_{P L}$ increases fastly with temperature increase. Differently, for sample B the $\mathrm{FWHM}_{P L}$ grows fast only for temperatures $\mathrm{T} \gtrsim 20 \mathrm{~K}$. 


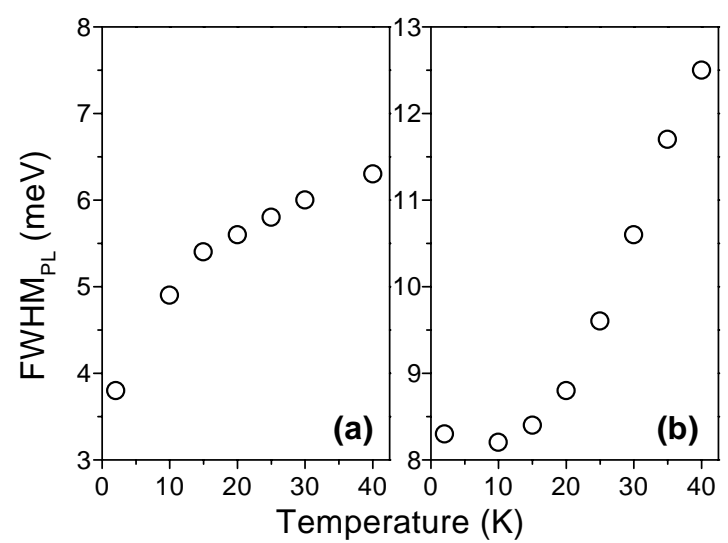

Figure 3. PL FWHM as a function of the temperature for samples (a) A and (b) B.

\section{Discussion}

We discuss the experimental results presented in the previous section based on a qualitative model that takes into account the interaction of the photogenerated carriers with the fluctuations of the confinement potential. The exciton energy level depends on $\mathrm{L}_{Q W}$ as well as on the QW depth, parameters that define the confinement potential in the growth direction ( $\mathrm{z}$ axis). However, variations in $\mathrm{L}_{Q W}$ and in the chemical composition of the barrier material along the plane perpendicular to the growth direction (xy plane) lead to fluctuations of the confinement potential along this plane. Since the variations in the $\mathrm{L}_{Q W}$ and in the chemical composition are random, the fluctuation magnitude is statistically distributed. Such a random potential fluctuation is sketched in Fig. 4. The exciton energy level is locally dependent on the full confinement potential. Since the PLE spectrum reflects the absorption process, there is a direct relationship between the line width of the PLE resonances and the intensity of the confinement potential fluctuations [3].

Before recombining, the photogenerated excitons loose their excess energy and in this process they feel the variation of the confinement potential. According to the energy excess and the magnitude of the potential variation, the excitons will relax either to local minima or to the absolute minimum of the confinement potential via phonon emission before radiative recombination. Thus, the FWHM $P L$ of PL spectra reflects the distribution of excitons in the energy states generated by the potential fluctuations. The energy difference between PLE and PL peaks, the Stokes shift, represents then the average of the difference between the energy of photocreated and minimum localized excitons. The higher the SS, the higher the confinement potential fluctuations magnitude.

The exciton dynamics is determined by the amount of thermal energy $(\mathrm{kT})$ and the magnitude of the lo- cal potential. With the increase of $\mathrm{kT}$ the exciton in a given potential minimum can jump to a deeper one or eventually recombine from the higher energy level. The relaxation to deeper minima causes the displacement of the PL peak towards lower energy values. Such a displacement is not observed in sample A [Fig. 2(a)], but is evident in sample B [Fig. 2(b)], which shows that the exciton relaxation is incomplete in sample $\mathrm{B}$ for low temperatures. In fact, the potential fluctuation magnitude should be higher in sample B than in sample A due to the thinner $\mathrm{L}_{Q W}$. Also in Fig. 2 we can see that the SS decreases in the higher temperature region. This is expected since the two-dimensional density of states is higher than the localized density of states generated by the potential fluctuations. In the same way, we can see in Fig. 3 that the $\mathrm{FWHM}_{P L}$ presents different behaviour for samples A and B. In the second one the curve goes through a shallow minimum in the low temperature region $(\mathrm{T} \lesssim 20 \mathrm{~K}$ ) before it starts to increase for increasing temperature, as observed for sample A. The comparison of the $\mathrm{FWHM}_{P L}$ behaviour then reinforces the previous conclusion that in samples with smaller $\mathrm{L}_{Q W}$ excitons are more sensitive to the interface disorder. Finally, the reduction of the intensity of the heavy hole (e1-hh1) PLE resonance for sample B relative to sample A, as shown in Fig. 1(b), is also a direct consequence of the larger $\mathrm{L}_{Q W}$ of sample $\mathrm{A}$, in agreement with Jahn et al. interpretation [5].

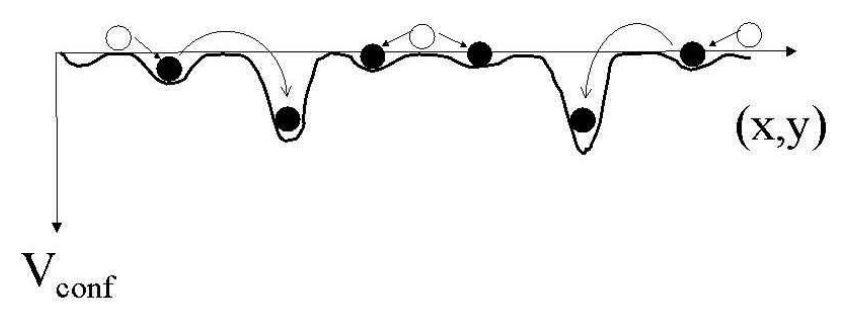

Figure 4. Sketch of the confinement potential $\mathrm{V}_{\text {conf }}$ along the xy plane of the QW. Open circles represent free excitons. Arrows indicate the relaxation processes to the local or absolute potential minima. Full circles represent excitons still localized at these minima.

\section{Summary}

In this work we report a comparative study of optical properties of two InGaP/GaAs quantum wells with same growth conditions but different $\mathrm{L}_{Q W}$. The peak position of PL and PLE, and the FWHM $_{P L E}$ and $\mathrm{FWHM}_{P L}$ as a function of temperature are presented. The different behaviour of these parameters for the two samples can be described by a qualitative model that accounts for the interaction of photogenerated excitons with the in-plane potential topography 
related to the interface disorder. The confinement potential fluctuations present minima that capture excitons, and prevent the complete relaxation of the excitonic population. From the global analysis of the data we see that anomalous temperature dependencies are more pronounced in the sample with smaller $\mathrm{L}_{Q W}$, in which excitons are more sensitive to the interface disorder.

\section{Acknowledgements:}

The authors gratefully acknowledge financial support from FAPESP, CAPES and CNPq.

\section{References}

[1] J. Singh, "Properties of Semiconductors and their Heterostructures", McGrawHill (London 1993).

[2] M. Grundmann, D. Bimberg, Phys. Rev. B38, 13486 (1988).

[3] M. Grassi Alessi, F. Fragano, A. Patanè, M. Capizzi, E. Runge, and R. Zimmermann, Phys. Rev. B61, 10985 (2000).

[4] G. Bastard, "Wave Mechanics Applied to Semiconductor Heterostructures", Les Éditions de Physique (Paris 1988).

[5] U. Jahn, M. Ramsteiner, R. Hey, H. T. Grahn, E. Runge, and R. Zimmermann, Phys. Rev. B56, R4387 (1997). 